MATEC Web of Conferences 44, 01082 (2016)

DOI: $10.1051 /$ matecconf/20164401082

C Owned by the authors, published by EDP Sciences, 2016

\title{
One Kind of Routing Algorithm Modified in Wireless Sensor Network
}

\author{
Ni Ni Wei ${ }^{1, a}$, Yi Song ${ }^{2}$ \\ ${ }^{1}$ Department of computer science\& Information Engineering, Wuhan Bioengineering Institute, Wuhan 430415, China \\ ${ }^{2}$ Department of computer science\& Information Engineering, Wuhan Bioengineering Institute, Wuhan 430415, China
}

\begin{abstract}
The wireless sensor networks are the emerging next generation sensor networks, Routing technology is the wireless sensor network communication layer of the core technology. To build reliable paths in wireless sensor networks, we can consider two ways: providing multiple paths utilizing the redundancy to assure the communication reliability or constructing transmission reliability mechanism to assure the reliability of every hop. Braid multipath algorithm and ReInforM routing algorithm are the realizations of these two mechanisms. After the analysis of these two algorithms, this paper proposes a ReInforM routing algorithm based braid multipath routing algorithm.
\end{abstract}

Key words: wireless sensor network; routing protocol; braid multipath; ReInforM routing

\section{INTRODUCTION}

Wireless sensor networks are the emerging next generation networks, which develop on the basis of the new network in the digital circuitry, wireless communications, micro-electromechanical systems and other technologies has become a hot research field.Hundreds of low cost, low power, small size, multifunctional sensor nodes in sensor networks, in the military and national defense, environmental monitoring, biomedical, smart homes, remote monitoring and other dangerous areas of the region has potentialuse value and broad application prospects [1].

For many sensor network applications with high reliability of data transmission requirements, its implementation depends mainly on the route chosen and reliable, so reliable routing protocol is particularly important.Wireless sensor network routing technology is the core technology of communications layer [23].Responsible for routing protocol data packets transmitted from the source node to destination node through the network to achieve in the sink node and sensor nodes transmit data reliably.

\section{2 sensor network architecture}

Wireless sensor network is deployed in the monitoring area by the large number of lowcost micro sensor nodes, which is the formation of a multi-hop ad hoc networks through wireless communication.Sensor networks is the integration of monitoring, control and wireless communication network system, its structure is shown in Figure 1, sensor network consists of sensor nodes, aggregation nodes, task management nodes and other components.Sensor nodes deployed in the designated monitoring area, the sensor nodes to monitor the data along to other sensor nodes hop by hop transmission, transmission of monitoring data in multiple nodes can be handled through the multi-hop routing to the sink node after, and finally through the Internetand satellite to reach management node, the user through the management node sensor network configuration and management, releases monitoring mission and the collection of monitoring data.

Wireless sensor network has the following characteristics: dense, and the large number of nodes; the location of sensor nodes is basically fixed; due to their own environmental impact of energy depletion and prone to failure node; environmental interference and node failures likely to cause changes in network topology;

\footnotetext{
a email: wnn19821024@163.com
} 
sensor node energy, processing power, storage capacity and communication bandwidth are very limited.Wireless sensor networks for these characteristics, the network protocol must be able to efficiently use energy and the local network information to be able to choose the right based on the path. This article does not intersect the path based on multi-path routing mechanism described, and put forward their views.

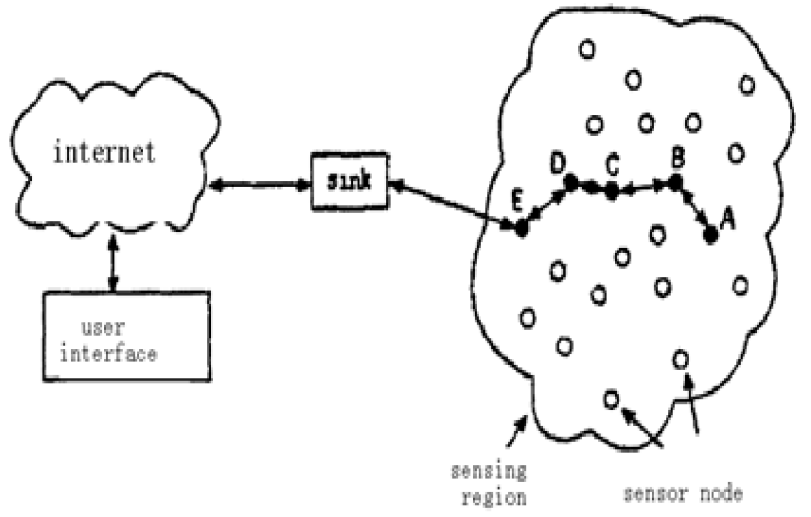

Figure 1 The network structure

\section{Multi-path based on the RelnforM winding route}

\subsection{Multi-path routing mechanism for winding}

In sensor networks, the introduction of multi-path routing is to increase the reliability of data transmission and implementation of network load balancing .In disjoint multipath, the alternate path may be much longer than the main path, for the introduction of spiral multipath (braid multipath) concept.Multi-path winding path to overcome the main problem of single node failure. The winding path is the ideal multi-winding path by the formation of a group.A winding path that corresponds to a node on the main path, not including the nodes in the network, the formation of the source node to destination node from the optimized backup path.Winding path as the primary path of a backup path. The main path, each node has a corresponding winding path, which constitutes a winding path from the source node to destination node more than the winding path. Obviously, such an alternate path by intersecting with the main path. Proposed a multi-path routing mechanism that preestablishment and maintenance of a path, do not need periodic flooding to be able to recover the data source node to sink node of the transmission path [3]. The basic idea is :First, build from the data source to the sink node of the main path, and then create a number of alternate paths; data transmission through the main path, while low-speed data transmission using alternate path to maintain the effectiveness of the path; When the primary path fails. When the path from the alternate path selected as the new sub-optimal path.

For the establishment of multiple paths method [4] proposed a multi-path disjoint (disjoint multipath) and wound multi-path (braid multipath) two algorithms is disjoint from the source node to destination node of any two pathsnodes do not intersect.Build process: first through the main path of convergence node enhanced information to establish the primary path; and then send the second best path to enhance the information to suboptimal node $\mathrm{A}$, node $\mathrm{A}$ and then choose their own optimal node $\mathrm{B}$, the second-best path to enhance the information passed on.If the primary path $\mathrm{B}, \mathrm{B}$ sends back information to enhance the negative $A$, A node to the second-best second-best path to enhance information transfer; if B is not the main path, then the B sub-optimal path to pass on increased information, until the construction of a secondoptimal path, according to the same methods, It may continue to construct a sub-optimal path to the next.

In disjoint multipath, the alternate path may be much longer than the main path, for the introduction of spiral multi-path (braid multipath) concept.Multi-path winding path to overcome the main problem of single node failure.The winding path is the ideal multi-winding path by the formation of a group.A winding path that corresponds to a node on the main path, not including the nodes in the network, the formation of the source node to destination node from the optimized backup path.Winding path as the primary path of a backup path.The main path, each node has a corresponding winding path, which constitutes a winding path from the source node to destination node more than the winding path.Obviously, such an alternate path by intersecting with the main path.

In ideal winding path, the nodes need to know the global network topology.A local multi-path generation algorithm winding as follows: the establishment of the main path, the primary path for each node (except the source node and the nodes near the source) should be enhanced alternate path to send information to their suboptimal node (denoted as A), the second best again to find the optimal node node (denoted as B) increased dissemination of information on the alternate path.If the node B is not the main path,It will continue to spread its optimal node, until the intersection with the main path to a new backup path.

In the two multi-path generation algorithm, the alternate paths have different priorities. When the primary path fails, the second best path will be activated to become the new primary path.

\subsection{RelnForM the routing mechanism}

In the sensor network, sensor nodes is the data source, the monitoring data sent to the sink node.ReInForM (Reliable Information Forwarding Using Multiple Paths) route starting from the data source node, 
consider the reliability, quality of communication and sensor node to sink node hop count to determine the number of required transmission path, and the number of nodes and the corresponding next hop nodeto achieve data transmission to meet reliability requirements.

In ReInForM routing, defines a reliability parameters, wit $\mathrm{h}$ a positive number $r_{s}$ indicates less than one. The argum ent that the system requirements for the data source to sen $\mathrm{d}$ data packets to the sink node probability of success.Eac $\mathrm{h}$ node knows its neighbor nodes to the channel quality, $\mathrm{u}$ sing the channel error rate $e_{s}$ that is less than one positive.

Suppose each node to all neighbors of quality is the same. Sensor nodes through the following mechanisms to the $\mathrm{s}$ ink node knows the number of hops hs: the sink nod e periodically broadcast routing updates, which includes a number of hops to the sink node dom ain, node routing update information, the infor mation in the number of hops to the sink node.

According to the data source node $r_{s}$ 、 $e_{s}$ and $h_{s}$ three parameters, we need to decide how many paths to forward data packets to ensure reliability. As $e_{s}$ to a link error rate, for the data source node, After $h_{s}$ the jump node packet arrival probability of convergence $\left(1-e_{s}\right)^{h_{s}}$, After $\mathrm{p}$ after the packet path can not reach the probability of convergence node $\left[1-\left(1-e_{s}\right)^{h_{s}}\right]^{p}$, so the success of the source node needsnumber of transmission path $\mathrm{p}$ can be calculated by the following formula:

$\mathrm{p}\left(r_{s}, e_{s}, h_{s}\right)=\frac{\lg \left(1-r_{s}\right)}{\lg \left(1-1-\left(1-e_{s}\right)^{h_{s}}\right)}$

Note: The following formula, $\mathrm{P}$ represents the source of all nodes required the number of successful transmission path; ${ }^{r}$ represents the system requirements for the data source to send data packets to the sink node probability of success; ${ }^{e_{s}}$ represents the channel error rate; ${ }^{h}$ represents the said he hops to the sink node of the $h_{s}$.

If $p$ is greater than the number of successful data transmission path to the neighbor data source node number, you need to send multiple copies of some neighbors to copy data to meet the reliability requirements.

Data source node needs to calculate the number of forwarding path after selecting the next hop neighbor nodes, and assign the appropriate path forward.According to a sink node hops, the source node to neighbor nodes into three categories [5-7]: with their hops to the same cluster node, the node, than their less number of hops to the sink node one, node, and to the sink node than theirmore than one node. These three nodes were used. First, select the source node of a next hop node as the default, the default next hop to forward data probability data node is one. Since the source node to the default next hop node sends a success rate of the data, this path is equivalent to the success of Article forwarding path.If greater than or equal to the equation (1) calculated the number of paths, indicating that the source node only need to default the next hop node can forward data to meet the reliability requirements.Otherwise, it requires additional forwarding nodes required number of additional paths:

$$
\mathrm{p}\left(r_{s}, e_{s}, h_{s}\right)=\frac{\lg \left(1-r_{s}\right)}{\lg \left(1-1-\left(1-e_{s}\right)^{h_{s}}\right)}-\left(1-e_{s}\right)
$$

First select additional nodes from the path of the $\mathrm{H}^{-}$, Only when the value of $\mathrm{p}$ is calculated greater than the number of nodes $H^{-}$in time accordance with the formula (2), need to select nodes from $H^{0}$; If the $\mathrm{p}$ value is greater than the sum of the number of nodes $H^{-}$and $H^{0}$, At this point need to select nodes from $H^{+}$. Each node in the collection have been selected as the source node to create a certain number of paths, To ensure that the path of all

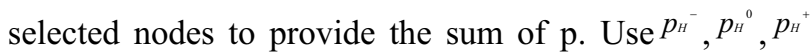
set that collection $H^{-}, H^{0}, H^{+}$, was selected as the next hop node need to create a number of paths the source node, Set $H^{-}, H^{0}, H^{+}$, in the order of selected nodes $N_{H^{-}}, N_{H^{+}}, N_{H^{0}}$, There have the formula (3)

$$
N_{H}{ }^{-} \cdot p_{H}{ }^{-}+N_{H}{ }^{0} \cdot p_{H}{ }^{0}+N_{H}{ }^{+} \cdot p_{H}{ }^{+}=p
$$

According to the following Proportion with $p_{H}{ }^{-}, p_{H}{ }^{0},{p_{H}}^{+}$:

$$
p_{H}{ }^{-}=\frac{p{ }^{0}}{1-e_{S}}=\frac{p_{H}{ }^{+}}{\left(1-e_{s}\right)^{2}}
$$

Select $H^{0}$ or $H^{+}$node as the next hop node; rather than repeat the selection $H^{-}$nodes in order to maintain the balance network load.

\subsection{Multi-path algorithm to improve the winding}

From the above detailed description we can see: many winding paths and disjoint multipath compared to the advantage of reducing the length of the alternate paths to improve the efficiency of data transmission, but the reliability is decreased.For more winding path, if the primary path and alternate path exists for a certain period of intersecting paths, when the intersecting paths fails, after their main path and alternate path will fail, if all paths have been intersecting with a path, andthis side of the path fails, the transmission will be terminated, only 
to re-establish a good network until the new primary path and alternate path can continue to transmit data.However, disjoint multipath algorithm, data transmission failure occurs only in the main path and a number of alternate paths at the same time fails.ReInForM routing mechanism has the advantage of the quality of communication channel through the transmission path and the path calculation of the number distribution, improve the reliability of data transmission and network load balancing, the energy limited by shortcomings in the face of such a routing mechanism for sensor networks toP path while transmission, energy will be costly. Therefore, it can improve the way we propose a route and wound ReInForM and combine multi-path algorithm: multi-path based on the ReInforM winding route, specifically described as follows:

Step 1: the first to use multi-pass algorithm winding aggregation node and between the source node $\mathrm{n}$ be a main path and alternate paths.

steset up $r_{s}=1, e_{s}=0$, Primary path used for data transmission.

Step 2: When the primary path fails, data transmission using ReInForM routing, set up ${ }_{s}$ The reliability of the system parameter values themselves, ${ }_{s}$ The channel error rate,

Step 3: At the same time, by priority level of the $n$ bar, choose an alternate path as a new path connecting the main path, if there is no such path, you need to use step 1 to re-establish the primary path and alternate path, and run step 2 .

\section{Conclusion}

In this paper, two multi-path routing algorithm and ReInforM described and analyzed, and on this basis, considering the advantages and disadvantages of each proposed a multi-winding path ReInforM based routing mechanism, this improved use of wound multi-path routing mechanismalgorithm for establishing a primary path and a number of alternate paths, when the main path in a normal state, only one main path used for data transmission, more than ReInforM multi-path routing algorithm to transfer data at the same time save a lot of energy, which energy Shousensor network is very limited sense.Failure at the main path routing to transfer data using ReInforM ensure the reliability of wireless sensor networks.Equations should be centred and should be numbered with the number on the right-hand side.

Acknowledgments. This paper is supported by Hubei Provincial Education projects (No.B2014160) and Wuhan Education Projects Foundation(No.2014217) and Wuhan Education Projects Foundation(No.2009k101)

\section{References}

1.Cui Li, Ju Hailing. And so on. Progress in wireless sensor networks [J]. Computer Research and Development, 2005 (1) :163-174.

2.Yaping LI.Analyze and Implementation of a New Route Protocol Based on MSAODV[J]. Microcomputer Information2007(8-2): 225-22

3.Qiangli Zhao, Ming Xu.Analysis and Comparison of Routing Protocols for Wireless Sensor Networks[J] Computer Science 2009.2(36):35-41.

4. D.Y Zhang, G.Q Ji. "Research on DSR-Based Energy Routing Protocols for Ad Hoc Networks". Computer Technology and Development. Vol.19,No.8,pp.1519,2009.

5. C.X Wu, L.Z Song, J.B Wang.“DSR ProtocolBased on Congestion Control and EnergyBalance Strategy".Contro 1 Engineering of China.VOL.17, NO 2,PP.260-263,2010. 6.Ahmad.S,Awan.I,Waqqas.A,et al. "Performance analys is of DSR\& extended DSR protocols". Proc.Kuala Lump ur: the Second Asia InternationalConference on Modeling \& Simulation, 2008.

7.weinini,songyi.A Reputation of Node Computation Mo del-based EXCHANG Inquiry Scheme in Ad Hoc Net wo rks[J].Experimental Technology and Management,2013 (4):96-98 